\title{
The physiological and perceptual demands of running on a curved non- motorised treadmill: Implications for self-paced training
}

\author{
Patrick P.J.M. Schoenmakers, Kate E. Reed
}

\section{Abstract \\ Objectives}

To compare physiological and perceptual response of running on a curved non-motorized treadmill (cNMT) with running on a motorized treadmill (MT), and to determine the running velocity at which a physiological response $\geq 90 \% \mathrm{VO}_{2}$ max was elicited.

\section{Design \& methods}

13 trained male runners (mean $\pm S D$; $36 \pm 11$ years, $1.80 \pm 0.06 \mathrm{~m}, 70 \pm 4 \mathrm{~kg}, \mathrm{VO}_{2} \max : 57.3 \pm 3.5 \mathrm{~mL}$ $\mathrm{kg}^{-1} \mathrm{~min}^{-1}$ ) performed an incremental running test on a MT to determine $\mathrm{VO}_{2}$ max and the accompanying maximum velocity (Vmax). Participants first completed a familiarization session on the cNMT. Next, participants ran for $4 \mathrm{~min}$ at five/six progressively higher velocities (40-90\% Vmax). These runs were completed on the cNMT and MT in two separate visits in a randomized and counterbalanced order.

\section{Results}

No participant was able to complete the 4 min run at $80 \%$ Vmax on the cNMT. Running on the cNMT elicit a higher relative oxygen uptake $\left(\% \mathrm{VO}_{2} \max \right)$ across all velocities compared to the MT (32.5 \pm $5 \%, p<0.001$, ES $3.3 \pm 0.9)$, and was accompanied by significantly higher heart rates $(16.8 \pm 3 \%, p<$ 0.001 , ES $3.4 \pm 1.5)$, an altered cadence $(2.6 \pm 0.7 \%, p<0.001$, ES $0.8 \pm 0.3)$ and ratings of perceived exertion $(27.2 \pm 5 \%, p<0.001$, ES $2.3 \pm 0.6)$. A less efficient running economy was evident when running on the cNMT $(+38.4 \pm 16 \%, p<0.001$, ES 2.73). Individual $(n=9)$ linear interpolation predicted an exercise intensity of $90 \% \mathrm{VO}_{2}$ max was achieved in the non-motorized condition when running at $62.1 \pm 3.5 \% \operatorname{Vmax}\left(\mathrm{R}^{2}=0.986 \pm 0.01\right)$, which was lower than $\mathrm{MT}$ run in which $90 \% \mathrm{VO}_{2}$ max was achieved at $81.4 \pm 5.6 \% \operatorname{Vmax}\left(R^{2}=0.985 \pm 0.02 ; 29.8 \pm 8 \%, p<0.001\right.$, ES 3.87).

\section{Conclusions}

Running on the cNMT has higher physiological and perceptual demands and increases cadence.

\section{Keywords}

Non-motorised treadmill; Running; HIIT; Self-paced training 


\section{Introduction}

Treadmills are an indispensable piece of laboratory equipment, and have become a key piece of exercise testing and training equipment. They are considered a valid measure of outdoor running performance, as evidenced by Jones \& Doust, ${ }^{1}$ who showed that the oxygen uptake $\left(\mathrm{VO}_{2}\right)$ during overground running and running on a motorized treadmill (MT) was strongly correlated with the use of a $1 \%$ treadmill grade. However, when performing a running task on a MT, moment-to-moment changes in velocity are not possible due to the fixed belt speed, and changes in velocity are controlled by an external motor which further requires a manual action to be changed. ${ }^{2,3}$ With the change of velocity controlled 'externally of the runner', deciding to change the running velocity requires a conscious decision by the runner. It is however suggested that the regulation of intensity during endurance exercise occurs unconsciously, based on live interactions with the environment and by both central and peripheral control mechanisms, ${ }^{4,5}$ and therefor the ecological validity of MT running may be questionable.

Recently, it has been argued that athletes measure and pace their work in training sessions in general, and in high intensity interval training (HIIT) specifically, on ratings of perceived exertion (RPE) and accumulated fatigue. ${ }^{6}$ This 'iso-effort' approach is in sharp contrast with protocols often used in lab based experiments, in which responses to predefined exercise intensities are studied. In self-paced HIIT, the maximum sustainable intensity is employed for a set number of work intervals of fixed durations. Athletes can then self-regulate their exercise intensity, based on their knowledge of the total volume of the session, the memory of similar events, as well as feedback from external and internal receptors. ${ }^{5,6}$ It is suggested that athletes should spend at least several minutes per HIIT in their 'red zone', which refers to the intensity domain close to their maximal oxygen uptake and heart rate $\left(\geq 90 \% \mathrm{VO}_{2}\right.$ max and HRmax respectively) ${ }^{7,8}$ While self-paced HIIT has been addressed recently in cycling, ${ }^{6,9}$ there is a paucity of research exploring the use of self-paced HIIT in running exercise.

Previously, the acute physiological responses to self-paced running HIIT protocols of varying work durations and/or recovery durations have been studied. ${ }^{10,11,12}$ However, in these studies participants ran on a MT and the velocity could be increased or decreased via a hand signal to the test administrator controlling the treadmill, highlighting the conscious external decision making process required. ${ }^{10,11,12}$ Standard MTs do not allow to study the quick and frequent adjustments in running velocities that occur during self-paced exercise. ${ }^{13}$ Non-motorized treadmills (NMT) on the other hand, are participant driven and allow runners to self-select their pace and dictate the speed of the treadmill belt with every step, which makes the overall locomotion more consistent with outdoor running. ${ }^{2,3}$ Previously, a commercially available curved NMT ((cNMT); Woodway Curve XL, Woodway, Waukesha, USA) demonstrated good reliability and validity for the assessment of $\mathrm{VO}_{2} \mathrm{max},{ }^{14}$ endurance performance, ${ }^{3,15}$ sprint $^{16}$ and repeated sprint intervals. ${ }^{17}$ To evaluate the potential use of the cNMT for self-paced HIIT sessions, it is important to understand the physiological responses associated with running on the cNMT. The aim of this study therefore was to determine the physiological and perceptual demands of running on a cNMT over a range of velocities commonly used in training and races of trained runners, and compare these to the demands of running on a MT set to a $1 \%$ gradient. The second aim was to determine at which running velocity a physiological response $\geq 90 \% \mathrm{VO}_{2}$ max was elicited on both the cNMT and MT. Trained club level runners were used in this study as they would likely be more attuned to internal pacing queues, and be able to maintain high workloads for sufficient time for reliable measures to be taken. It was hypothesized that the physiological demands of running on the cNMT would be higher than on a MT at any given velocity, since the curved design introduces a slight incline to the front aspect of the treadmill, which in theory demands higher energy expenditure.

\section{Methods}

Participants visited the sport and exercise science laboratory on 4 different occasions over a twoweek period, with visits separated by at least $48 \mathrm{~h}$. During their first visit, participants performed an incremental running test to voluntary exhaustion on a MT (Pulsar 3p, H/P Cosmos, NussdorfTraunstein, Germany) to determine $\mathrm{VO}_{2} \mathrm{max}$ and the associated running intensity (peak treadmill velocity $(\operatorname{Vmax}))$. On the second visit, participants performed the experimental running protocol (detailed below) on the cNMT as a familiarisation session (cNMTfam). Two comparative experimental sessions on the cNMT (cNMTrun) and MT (MTrun) in the third and fourth visit were performed in a counterbalanced and randomized order. All visits were completed at the same time of the day $( \pm 1 \mathrm{~h})$. 
Participants were asked to refrain from any strenuous exercise or alcohol consumption in the preceding $24 \mathrm{~h}$, and from caffeine and food consumption, 4 and $2 \mathrm{~h}$ before the start of the test, respectively. Two factory calibrated treadmills were used in this study: a standard motorised treadmill, and a non-motorised treadmill with a curved surface. Accuracy of both treadmill's velocity measures were verified using a video camera and found to be within $<1.1 \%$ of the described speed. Participants were asked to report for testing wearing the same footwear on each visit.

Thirteen club level male runners (mean $\pm S D, 36 \pm 11$ years; $1.80 \pm 0.06 \mathrm{~m} ; 70 \pm 4 \mathrm{~kg}$; $\mathrm{VO}_{2} \max : 57.3 \pm$ $3.5 \mathrm{~mL} \mathrm{~kg}-1 \mathrm{~min}-1$; Vmax: $5.0 \pm 0.2 \mathrm{~m} \mathrm{~s}-1)$ participated in this investigation. No participant had prior experience with (curved) NMT running. Prior to their active participation, all participants provided voluntary written informed consent. The study received approval from the local ethics committee (University of Essex, UK) and was conducted in accordance with the Declaration of Helsinki.

During their first visit, participants performed an incremental running test on the MT, with the gradient set at $1 \%$. This test started at $2.22 \mathrm{~m} \mathrm{~s}^{-1}$, which was increased by $0.28 \mathrm{~m} \mathrm{~s}^{-1}$ each minute until participants reached volitional exhaustion or when one of the following criteria was met: (1) maximal heart rate at least equal to $90 \%$ of the age-predicted maximum; (2) respiratory exchange ratio (RER) $\geq 1.1$; (3) stable oxygen consumption despite increased intensity. ${ }^{18} \mathrm{VO}_{2}$ max was defined as the highest 30 -s averaged $\mathrm{VO}_{2}$ collected during the incremental test. HRmax was defined as the highest value obtained at the end of the test. Vmax was defined as the highest velocity that could be maintained for a complete minute, or as the velocity of the last complete stage added to the completed fraction of an incomplete stage. Vmax was calculated according to the equation Vmax = $\mathrm{Vcomp}+\left(0.28 \mathrm{~m} \mathrm{~s}^{-1} \times \mathrm{t} / 60\right)$, in which Vcomp is the velocity of the last completed stage and the time in seconds sustained during the final incomplete stage.

In the familiarisation and comparative experimental runs, participants were required to run for 4 min at five different individualized velocities $(40 \%, 50 \%, 60 \%, 70 \%, 80 \%$ Vmax; velocity range $[2.0 \pm 0.1-4.0$ $\left.\pm 0.2 \mathrm{~m} \mathrm{~s}^{-1}\right]$ ), with $3 \mathrm{~min}$ passive recovery between the $4 \mathrm{~min}$ runs. A subset of eight participants volunteered to complete a further (6th) running bout at $90 \%$ Vmax on the MT (velocity: $4.5 \pm 0.2 \mathrm{~m}$ $\mathrm{s}^{-1}$ ). In cNMTfam and cNMTrun, participants were instructed to monitor their speed on the treadmill's LCD screen and maintain it as close to the prescribed speed as possible. ${ }^{3}$ Verbal cues to do so were provided if necessary. Average oxygen consumption, heart rate, RER and running cadence were determined during the last minute of each stage, together with overall RPE on the standard Borg scale. $^{19}$

Running economy was calculated using the averaged $\mathrm{VO}_{2}$ and $\mathrm{RER}$ from the final min of the runs conducted at $50 \% \operatorname{Vmax}\left(2.5 \pm 0.09 \mathrm{~m} \mathrm{~s}^{-1}\right)$. This speed was selected as 11 subjects completed cNMTrun and MTrun at this speed with an RER $<1.0$. Running economy was expressed as gross oxygen unit cost ( $\left.\mathrm{mL} \mathrm{kg}^{-1} \mathrm{~km}^{-1} 1\right)$, as well as a gross caloric unit cost (the energy required to cover a given distance; $\mathrm{kcal} \mathrm{kg}^{-1} \mathrm{~km}^{-1}$ ). The gross caloric unit cost was calculated as described by Fletcher et al., ${ }^{20}$ in which the averaged RER was used to determine the caloric equivalent of $\mathrm{VO}_{2}$.

During the incremental running test, cNMTfam, and the two comparative experimental runs, heart rate and running cadence were measured continuously at $1 \mathrm{~Hz}$ using a Garmin heart rate monitor and a telemetric foot pod (910XT, Garmin Ltd., Schaffhausen, Switzerland). Respiratory parameters were measured breath by breath, using open circuit spirometry (Oxycon Delta, Jaeger, Höchberg, Germany). The gas analyser was calibrated prior to each test using room air and a calibration gas $\left(16.0 \% \mathrm{O}_{2}, 5.0 \% \mathrm{CO}_{2}\right)$. The physiological measures of both $\mathrm{VO}_{2}$ and $\mathrm{HR}$ were indexed for individual $\mathrm{VO}_{2} \max$ and $\mathrm{HRmax}\left(\% \mathrm{VO}_{2} \max\right.$ and \%HRmax respectively), to use these relative values as an insightful indicator of the relative exercise intensity, especially in the intense exercise domain in which $\mathrm{VO}_{2}$ is not expected to reach steady state. Running velocity in the cNMT trials was sampled at $4 \mathrm{~Hz}$, and was assessed in the accompanying product software.

Data were analysed using SPSS 23.0 (SPSS Inc., USA) and are presented as mean \pm SD. Differences in running velocities were compared between cNMTfam, cNMTrun and MTrun using repeated measures ANOVAs. A comparison between cNMTfam and cNMTrun was carried out to observe any training effects due to the novelty of this piece of equipment. Repeated measures ANOVAs were carried out to compare differences between conditions (cNMTrun vs MTrun) in $\% \mathrm{VO}_{2}$, $\mathrm{HR}, \mathrm{RER}$, running cadence and RPE for each velocity. In an attempt to determine the running velocity which elicited comparable exercise intensities between the CNMT and MT, data collected during $90 \%$ 
Vmax on the MT were compared with data collected during 70\% Vmax cNMT ( $\mathrm{n}=8$ participants) using paired t-tests. The running velocity at which the physiological response corresponded to $90 \%$ $\mathrm{VO}_{2} \max$ was determined individually, through linear interpolation for both treadmills. Standardized effect sizes are reported as Cohen's d. Qualitative interpretation of $d$ was based on the guidelines provided by Hopkins: $<0.2$ trivial; $0.20-0.59$ small; $0.6-1.19$ moderate; $1.20-1.99$ large; $\geq 2.00$ very large. The significance level of all tests was set at $<0.05$

\section{Results}

A summary of experimental variables is presented in Table 1. No participant was able to complete the 4 min running bout at $80 \%$ Vmax in cNMTfam or cNMTrun. No differences in running velocities were found between cNMTfam and cNMTrun $(40.2 \pm 0.8$ vs $40.3 \pm 0.8 ; 50.2 \pm 0.6$ vs $50.1 \pm 0.6 ; 60.2 \pm 0.7$ vs $60.2 \pm 0.7 ; 70.0 \pm 0.6$ vs $70.2 \pm 0.7$ for $40,50,60,70 \%$ Vmax respectively, $p>0.05$ ), or between cNMTrun and cMTrun. No learning effect was found of the familiarisation session, as no differences were apparent between cNMTfam and cNMTrun in any of the experimental variables.

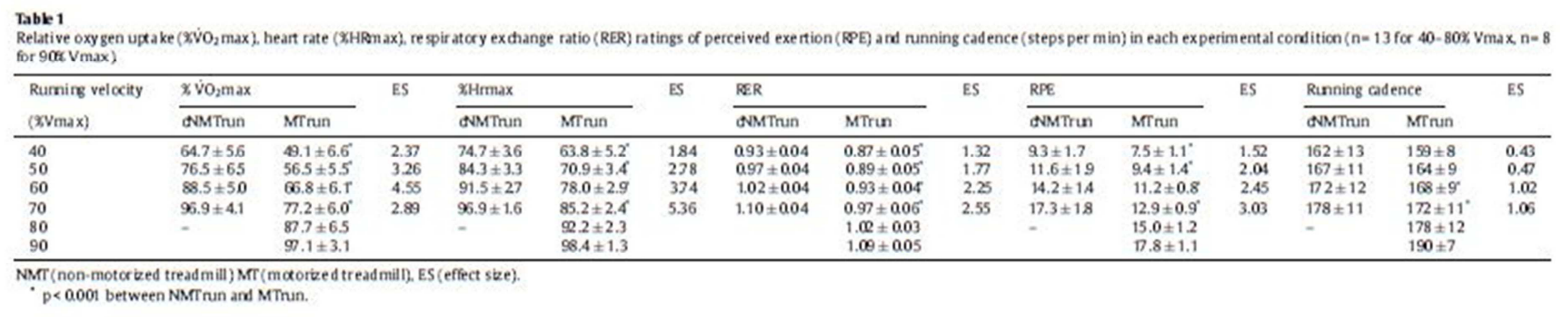

The average oxygen uptake in cNMTrun was significantly higher at all velocities compared to MTrun $(p<0.001)$. On average, across the four different velocities, the oxygen consumption was $32.3 \pm 4 \%$ higher in cNMTrun (see Table 1). The higher oxygen uptake was accompanied by significantly higher exercise heart rates $(+16.8 \pm 3 \%, p<0.001)$ and ratings of perceived exertion $(+27 \pm 5 \%, p<0.001)$. Running cadence was higher at all velocities in cNMTrun $(+2.4 \pm 0.8 \%)$, which reached statistical significance at $60 \%$ and $70 \% \operatorname{Vmax}(p<0.005)$. Differences in running economy were evident between both treadmills (see Table 2), with the economy in gross oxygen cost and in caloric cost being significantly lower in MTrun compared to cNMTrun $(-38.4 \pm 16 \%, p<0.001$, ES 2.73), indicating more economical running in MTrun.

Table 2. Runing Economy for each experimental treadmill, running on $50 \% \operatorname{Vmax}(n=11)$.

\begin{tabular}{llll} 
Running Economy: & cNMTrun & MTrun & ES \\
\hline Oxygen unit cost $\left(\mathbf{m L ~ O}_{\mathbf{2}} \mathbf{k g}^{-1} \mathbf{k m}^{-1}\right)$ & $279 \pm 37$ & $206 \pm 29^{*}$ & 2.73 \\
Caloric Unit Cost $\left(\mathbf{k c a l ~ k g}^{-1} \mathbf{~ k m}^{-1}\right)$ & $1.39 \pm 0.19$ & $1.01 \pm 0.14^{*}$ &
\end{tabular}

cNMT (curved non-motorized treadmill) MT (motorized treadmill), ES (effect size).

$\mathrm{p}<0.001$ between cNMTrun and MTrun.

Table 3 shows data comparing $70 \%$ Vmax cNMT and 90\% Vmax MT $(n=8)$. Apart from a significantly higher cadence in MTrun $(p=0.001)$, there were no differences in physiological or psychological responses. All thirteen participants reached an exercise intensity of $\geq 90 \% \mathrm{VO}_{2} \mathrm{max}$ in NMTrun. Linear interpolation of the available data in cNMTrun predicted an exercise intensity of $90 \%$ $\mathrm{VO}_{2} \max$ was achieved when running above $62.7 \pm 3.3 \% \mathrm{Vmax}\left(\mathrm{R}^{2}=0.986 \pm 0.01\right)$. Nine out of the thirteen participants reached $90 \% \mathrm{VO}_{2}$ max in MTrun, at a running velocity of $81.4 \pm 5.6 \% \mathrm{Vmax}\left(\mathrm{R}^{2}=\right.$ $0.985 \pm 0.02)$, which was significantly higher $(p<0.001$, ES 3.87). 
Table 3. Comparison of relative oxygen uptake $\left(\% \mathrm{~V}_{2} \max \right)$, heart rate (\%HRmax), respiratory exchange ratio (RER), ratings of perceived exertion (RPE) and running cadence (Steps per min) between $70 \%$ Vmax cNMTrun and $90 \%$ Vmax MTrun $(n=8)$.

\begin{tabular}{llll} 
& \multicolumn{2}{c}{ Running velocity } & \\
\cline { 2 - 3 } & $70 \%$ Vmax cNMTrun & $90 \%$ Vmax MTrun & 0.18 \\
\hline$\% \mathrm{VO}_{2} \max$ & $97.9 \pm 3.8$ & $97.1 \pm 3.1$ & 0.35 \\
$\% \mathrm{HRmax}$ & $97.0 \pm 1.5$ & $98.2 \pm 1.5$ & 0.45 \\
$\mathrm{RER}$ & $1.10 \pm 0.04$ & $1.09 \pm 0.05$ & 0.95 \\
$\mathrm{RPE}$ & $17.5 \pm 1.7$ & $17.8 \pm 1.2$ & 1.82 \\
Cadence & $182 \pm 6$ & $190 \pm 8^{*}$ &
\end{tabular}

cNMT (curved non-motorized treadmill) MT (motorized treadmill), ES (effect size).

$\mathrm{p}<0.01$ between $\mathrm{cNMT}$ and MT

\section{Discussion}

Non-motorized treadmills allow runners to adjust their running velocity subconsciously, consistent with outdoor running, and thus may be more appropriate apparatus to study self-paced training. This study aimed to (1) determine the physiological and perceptual demands of running on a curved NMT over a range of velocities, and (2) verify at which running velocity a physiological response $\geq 90 \% \mathrm{VO}_{2} \mathrm{max}$ was elicited.

When running on a NMT, participants must generate power to move themselves vertically and to propel the treadmill belt, ${ }^{21}$ which the manufacturer claims to result in a $30 \%$ higher caloric expenditure compared to running on a standard MT. Part of this higher energy expenditure may be due to a range of cNMT characteristics (e.g. a high mechanical resistance and rubber material of the treadmill belt), and may be inherent to the treadmill design. Findings of the current study support the manufacturers claim, as $\mathrm{VO}_{2}$ was, on average $32.2 \pm 4 \%$ higher in NMTrun across the different velocities.

Furthermore, an increase in caloric cost of $38.4 \pm 16 \%$ was evident when participants ran at $50 \%$ Vmax ( $1.39 \pm 0.19$ vs $1.01 \pm 0.14 \mathrm{kcal} \mathrm{kg}^{-1} \mathrm{~km}^{-1}$ for cNMT and MTrun respectively). These results are in line with Smoliga et al., ${ }^{22}$ who showed that walking $\left(1.34 \mathrm{~m} \mathrm{~s}^{-1}\right)$ and running $\left(2.24 \mathrm{~m} \mathrm{~s}^{-1}\right)$ on the cNMT elicits a greater physiological stimulus than that on MT. The running velocity of $2.24 \mathrm{~m} \mathrm{~s}^{-1}$ used by Smoliga et al. ${ }^{22}$ corresponds to $45 \%$ Vmax of the participants in the current study. This study aimed to evaluate the physiological responses to a broader range of (higher) running velocities, and, additionally, attempted to identify the running velocity that elicits an exercise intensity $\geq 90 \% \mathrm{VO}_{2} \mathrm{max}$ on both treadmills. Linear interpolation showed this intensity was achieved when running at $62.7 \pm$ $3.3 \%$ Vmax on the cNMT. Similar exercise intensity was reached in nine out of thirteen participants on the MT at $81.4 \pm 5.6 \%$ Vmax. The difference in \%Vmax for the nine participants that reached $\geq 90 \%$ $\mathrm{VO}_{2}$ max in both NMTrun and MTrun was $19.1 \pm 5.1 \%$. This is similar to the findings of Stevens et al. ${ }^{3}$ and Waldman et al., ${ }^{15}$ who both reported that $5 \mathrm{~km}$ running performance on the curved NMT was significantly slower compared to overground running (22\%)3 and MT running (24\%), 15 even though no differences in $\mathrm{VO}_{2}$ and exercise heart rates were found. In another recent study, Morgan et al. ${ }^{.4}$ observed a significant $15 \%$ lower Vmax on cNMT compared to a MT during an incremental running tests, where again, the participants were exerting the same exercise intensity in both tests. The large differences in running velocities while exercising at comparable exercise intensities highlights the disparity between the two treadmills.

The nature of the cNMT is such that users are required to run on an uphill gradient. To increase running velocity, participants position their feet closer to the front of the curved belt, which allows a 
greater contribution of vertical force to treadmill belt propulsion. Curved treadmill belts may facilitate a more natural gait pattern, allowing increased stride length and longer swing phase, which is observed with over-ground locomotion. Observational analysis by Smoliga et al., ${ }^{22}$ revealed that subjects contact the curved treadmill belt approximately at a five to ten degree incline above the horizontal and this angle decreases throughout the stance phase of the stride cycle. It has been suggested that runners always optimise their technique to minimise metabolic costs, and when an inclination is present, runners will modify mechanical variables to achieve optimal metabolic efficiency. ${ }^{23}$ Stevens et al., ${ }^{3}$ reported a change in running technique between overground and cNMT running. In overground running their subjects $(n=10)$ were classified as predominantly 'rear-foot strikers' $(9,1$ "mid-foot strike"), which in cNMT running changed to "mid-foot strike" (8, 2 'rear-foot strikers'). The change in running technique was further evident in changes in muscle recruitment patterns between cNMT and over-ground running, as they showed a decline in IEMG activity for tibialis anterior, vastus lateralis and rectus femoris in the former. ${ }^{3}$ The decrease in iEMG is most likely compensated with an increased EMG activity of the gluteus maximus and bicep femoris while running uphill. ${ }^{24}$ In line with previous research, an increase in energy expenditure, and running cadence was found in cNMTrun compared to MTrun during all experimental velocities, ${ }^{23,25}$ highlighting the uphill running character of the cNMT. Recently, several studies have examined the effects of a variety of uphill HIIT protocols, using repeated short (6-30 s) and/or long (3-5 min) work intervals on a variety of treadmill gradients. ${ }^{26,27}$ These studies showed improvements in various physiological, biomechanical, and neuromuscular parameters relevant to running performance, and provide support to incorporate uphill HIIT in the training programs of distance runners. Future research is needed to evaluate which treadmill gradient is most comparable to the curved design of the NMT, in aid to design the most appropriate training intervention. Self-paced uphill HIIT is a promising focus area for future research, and the cNMT allows evaluation of physiological responses in a well-controlled lab setting.

In the current study $\mathrm{VO}_{2}$ max and $\mathrm{Vmax}$ were determined using a traditional incremental running test, performed on a MT. The obtained $\mathrm{VO}_{2} \max$ was then used to compute and compare the relative oxygen uptake between cNMTrun and MTrun on different individualized running velocities. It is well known that $\mathrm{VO}_{2} \max$ is dependent on the physiological conditions present during an exercise protocol, ${ }^{18}$ and it has been shown that a self-paced $\mathrm{VO}_{2}$ max test performed on a NMT could possibly result in an elevated $\mathrm{VO}_{2}$ max. ${ }^{28}$ In fact, $\mathrm{VO}_{2}$ in three of the thirteen participants was higher in cNMTrun while running at $70 \% \operatorname{Vmax}$ than reached during their incremental running test. Conversely, Morgan et al., ${ }^{14}$ found no differences in attained $\mathrm{VO}_{2}$ max or maximum heart rate between MT and the cNMT. Thus, the increased $\mathrm{VO}_{2}$ in those few individuals in the current study may be attributed to the difference in bout duration between the experimental runs $(4 \mathrm{~min})$ and stage length in the incremental exercise test $(1 \mathrm{~min})$, rather than the different treadmills. $\mathrm{VO}_{2}$ continued to increase at $70 \% \mathrm{Vmax}$ in NMTrun, showing 'oxygen drift'. Oxygen drift is potentially caused by increased muscle fibre recruitment, changes in efficiency, body temperature and the increase of muscle fatigue over time, which all contribute to a larger amplitude in the slow component of $\mathrm{VO}_{2} \cdot{ }^{29}$ The increased contribution of this slow component of $\mathrm{VO}_{2}$ likely elevated the oxygen uptake in the 4 min run.

A limitation of this study is the inability to state the anaerobic contrition to metabolic work during the higher intensity intervals. When RER exceeds 1.0 the energetic cost of the exercise is more difficult to estimate, and it is not possible to compare energetic cost across individuals or across trials.

The results of the present study further information in the field of NMT running, by providing comparison velocities at which physiological workload is matched. Participants in the current study ran at higher and individualized velocities compared to previous studies, which yield new insights into the physiological and perceptual responses in the intense exercise domain. Only a subgroup $(n=8)$ opted to complete the $90 \%$ Vmax run, and this would have had implications related to statistical power.

\section{Practical applications}

- The cNMT can be a useful tool to study self-paced interval training. When prescribing exercise specialists often assign a specific (treadmill) velocity and duration as the primary training variables. Our data show that exercise prescriptions that are appropriate for overground or MT running may not be achievable on the cNMT because of the differences in energetic requirements. 
- Based on our results, it is advised to lower the running velocity by $20 \%$ when running on the cNMT to generate the comparable physiological stimulus.

- Running on the cNMT mimics uphill running, and therefore training adaptations may differ compared to over-ground or regular treadmill training.

\section{Author contributions}

PS and KR contributed to conception and design of the work. PS conducted the experiment, analyzed the data and wrote the first draft. Both authors were involved in further data analysis and drafting, and revized the manuscript critically for important intellectual content.

\section{References}

1. Jones AM, Doust JH. A $1 \%$ treadmill grade most accurately reflects the energetic cost of outdoor running. J Sports Sci 1996; 14(4):321-327.

http://dx.doi.org/10.1080/026404196367796.

2. Minetti AE, Boldrini L, Brusamolin L et al. A feedback-controlled treadmill (treadmill-ondemand) and the spontaneous speed of walking and running in humans. J Appl Physiol 2003; 95(2).

3. Stevens CJ, Hacene J, Wellham B et al. The validity of endurance running performance on the Curve 3n TMn non-motorised treadmill. J Sports Sci 2015; 33(11):1141-1148.

http://dx.doi.org/10.1080/02640414.2014.986502.

4. Smits BLM, Pepping G-J, Hettinga FJ. Pacing and decision making in sport and exercise: the roles of perception and action in the regulation of exercise intensity. Sports Med 2014; 44(6):763-775. http://dx.doi.org/10.1007/s40279-014-0163-0.

5. St Clair Gibson A, Swart J, Tucker R. The interaction of psychological and physiological homeostatic drives and role of general control principles in the regulation of physiological systems, exercise and the fatigue process - the Integrative Governor theory. Eur J Sport Sci 2017:1-12. http://dx.doi.org/10.1080/17461391.2017.1321688.

6. Seiler S, Jøranson K, Olesen BV et al. Adaptations to aerobic interval training: interactive effects of exercise intensity and total work duration. Scand J Med Sci Sport 2013; 23(1):7483. http://dx.doi.org/10.1111/j.1600-0838.2011.01351.x.

7. Midgley AW, McNaughton LR, Wilkinson M. Is there an optimal training intensity for enhancing the maximal oxygen uptake of distance runners?: empirical research findings, current opinions, physiological rationale and practical recommendations. Sports Med 2006; 36(2):117-132.

8. Buchheit M, Laursen PB. High-intensity interval training, solutions to the programming puzzle: Part I: cardiopulmonary emphasis. Sports Med 2013; 43(5):313-338. http://dx.doi.org/10.1007/s40279-013-0029-x.

9. Nicolò A, Bazzucchi I, Haxhi $\mathrm{J}$ et al. Comparing continuous and intermittent exercise: an "Isoeffort" and "Isotime" approach. Earnest CP, ed. PLoS One 2014; 9(4):e94990. http://dx.doi.org/10.1371/journal.pone.0094990.

10. Seiler S, Sjursen JE. Effect of work duration on physiological and rating scale of perceived exertion responses during self-paced interval training. Scand J Med Sci Sport 2004; 14(5):318-325. http://dx.doi.org/10.1046/j.1600-0838.2003.00353.x.

11. Seiler S, Hetlelid KJ. The impact of rest duration on work intensity and RPE during interval training. Med Sci Sports Exerc 2005; 37(9):1601-1607. 
12. Laurent CM, Vervaecke LS, Kutz MR et al. Sex-specific responses to self-paced, highintensity interval training with variable recovery periods. J Strength Cond Res 2014; 28(4):920-927. http://dx.doi.org/10.1519/JSC.0b013e3182a1f574.

13. Tucker R, Bester A, Lambert EV et al. Non-random fluctuations in power output during selfpaced exercise. Br J Sports Med 2006; 40(11):912-917. http://dx.doi.org/10.1136/bjsm.2006.026435, discussion 917.

14. Morgan AL, Laurent CM, Fullenkamp AM. Comparison of VO2 peak performance on a motorized vs. a nonmotorized treadmill. J Strength Cond Res 2016; 30(7):1898-1905. http://dx.doi.org/10.1519/JSC.0000000000001273.

15. Waldman HS, Heatherly AJ, Waddell AF et al. 5-km time trial reliability of a non-motorized treadmill and comparison of physiological and perceptual responses versus a motorized treadmill. J Strength Cond Res 2017; 1. http://dx.doi.org/10.1519/JSC.0000000000001993.

16. Gonzalez AM, Wells AJ, Hoffman JR et al. Reliability of the Woodway curve(TM) nonmotorized treadmill for assessing anaerobic performance. J Sports Sci Med 2013; 12(1):104108.

17. Sirotic AC, Coutts AJ. The reliability of physiological and performance measures during simulated team-sport running on a non-motorised treadmill. J Sci Med Sport 2008; 11(5):500509. http://dx.doi.org/10.1016/j.jsams.2007.04.008.

18. Poole DC, Jones AM. Measurement of the maximum oxygen uptake $\mathrm{VO}_{2 \max }: \mathrm{VO}_{2 \text { peak }}$ is no longer acceptable. J Appl Physiol 2017; 122(4):997-1002. http://dx.doi.org/10.1152/japplphysiol.01063.2016.

19. Borg G. Perceived exertion as an indicator of somatic stress. Scand J Rehabil Med 1970; 2(2):92-98.

20. Fletcher JR, Esau SP, MacIntosh BR. Economy of running: beyond the measurement of oxygen uptake. J Appl Physiol 2009; 107(6):1918-1922.

http://dx.doi.org/10.1152/japplphysiol.00307.2009.

21. De Witt JK, Lee SMC, Wilson CA et al. Determinants of time to fatigue during nonmotorized treadmill exercise. J Strength Cond Res 2009; 23(3):883-890.

http://dx.doi.org/10.1519/JSC.0b013e3181a04de9.

22. Smoliga JM, Hegedus EJ, Ford KR. Increased physiologic intensity during walking and running on a non-motorized, curved treadmill. Phys Ther Sport 2015; 16(3):262-267. http://dx.doi.org/10.1016/j.ptsp.2014.09.001.

23. Padulo J, Powell D, Milia R et al. A paradigm of uphill running. Seebacher F, ed. PLoS One 2013; 8(7):e69006. http://dx.doi.org/10.1371/journal.pone.0069006.

24. Wall-Scheffler CM, Chumanov E, Steudel-Numbers $\mathrm{K}$ et al. Electromyography activity across gait and incline: the impact of muscular activity on human morphology. Am J Phys Anthropol 2010; 143(4):601-611. http://dx.doi.org/10.1002/ajpa.21356.

25. Minetti AE, Moia C, Roi GS et al. Energy cost of walking and running at extreme uphill and downhill slopes. J Appl Physiol 2002; 93(3):1039-1046. http://dx.doi.org/10.1152/japplphysiol.01177.2001.

26. Barnes KR, Hopkins WG, McGuigan MR et al. Effects of different uphill intervaltraining programs on running economy and performance. Int J Sports Physiol Perform 2013; 8(6):639-647. http://dx.doi.org/10.1016/j.jsams.2012.11.035. 
27. Ferley D, Hopper D, Vukovich M. Incline treadmill interval training: short vs. long bouts and the effects on distance running performance. Int J Sports Med 2016; 37(12):958-965. http://dx.doi.org/10.1055/s-0042-109539.

28. Mauger AR, Metcalfe AJ, Taylor $L$ et al. The efficacy of the self-paced $V_{2} O_{2 m a x}$ test to measure maximal oxygen uptake in treadmill running. Appl Physiol Nutr Metab 2013; 38(12):12111216. http://dx.doi.org/10.1139/apnm-2012-0384.

29. Jones AM, Grassi B, Christensen PM et al. Slow component of VO2 kinetics. Med Sci Sport Exerc 2011; 43(11):2046-2062. http://dx.doi.org/10.1249/MSS.0b013e31821fcfc1. 